\section{Conhecimentos e práticas de promoção do aleitamento materno em Equipes de Saúde da Família em Montes Claros, Minas Gerais, Brasil}

\author{
Knowledge and practices in breastfeeding \\ promotion by Family Health teams in \\ Montes Claros, Brazil
}

1 Universidade Estadual de Montes Claros, Montes Claros, Brasil.

Correspondência A. P. Caldeira

Departamento de Saúde da Mulher e da Criança, Universidade Estadual de Montes Claros. Rua Monte Pascoal 225, Montes Claros, $M G$ 39401-347, Brasil. antonio.caldeira@unimontes.br

\begin{abstract}
The Family Health Strategy has been implemented in Brazil with the aim of reorganizing the health care system with an emphasis on promotion and prevention. Thus, encouragement for breastfeeding is one of the most important activities by family health care teams. The objective of this study was to assess knowledge and practices by family health care teams in relation to breastfeeding. In addition to a questionnaire on health professionals' characteristics, a specific knowledge test on breastfeeding was conducted with 41 physicians and nurses and another test on the same subject was given to 152 community health workers. Scores were above $80 \%$ on the advantages of breastfeeding in all the teams, but knowledge on practical issues concerning technique and management of the main problems encountered in breastfeeding was not uniform among the health professions. Nurses scored better than physicians, and community health workers had the lowest scores. Practical activities to promote breastfeeding were not developed uniformly among health care professionals. There is a need for systematic and ongoing training in breastfeeding management in the family health teams.
\end{abstract}

Breast Feeeding; Family Health Program; Primary Health Care; Health Promotion
Antônio Prates Caldeira 1 Gabriel Nobre de Aguiar 1 Weslane Almeida Cavalcanti Magalhães 1 Gizele Carmem Fagundes 1

\section{Introdução}

A estratégia de saúde da família deve assumir atividades preventivas como suas ações prioritárias. No âmbito da saúde materno-infantil, o incentivo ao aleitamento materno se apresenta como uma das principais ações para profissionais da atenção básica. O leite materno representa o melhor alimento para a criança nos primeiros meses de vida $1,2,3$. Todavia, a amamentação não é uma prática natural. Para melhoria dos seus índices faz-se necessário adequado aprendizado das mães com participação ativa dos profissionais de saúde, propiciando orientações e suporte oportunos para as gestantes e lactantes 4 .

Avaliar o conhecimento desses profissionais representa, pois, uma estratégia de reconhecimento do cenário de apoio à prática da amamentação. Estudo realizado com médicos, nos Estados Unidos, mostrou que, apesar destes reconhecerem seu potencial em influenciar positivamente o aleitamento materno, relatavam limitada capacitação específica para promoverem a prática 5 . Outros estudos apontam a situação de conhecimentos e práticas de outros profissionais de saúde em relação à amamentação e a relevância de treinamentos específicos na área 6,7,8.

O presente trabalho procurou investigar o nível de conhecimento dos profissionais de saúde das equipes de saúde da família e suas práticas em relação à promoção e apoio ao aleitamento materno. 


\section{Material e métodos}

A área do presente estudo foi a cidade de Montes Claros, ao Norte de Minas Gerais, Brasil. Com aproximadamente 340 mil habitantes, o município contava, na época desta pesquisa, com 29 equipes completas da estratégia de saúde da família (sendo três em área rural), totalizando 58 profissionais de nível superior e 172 de nível médio, que ofereciam assistência para, aproximadamente, $30 \%$ da população. A coleta de dados foi realizada entre outubro e dezembro de 2005.

Não houve cálculo amostral para seleção do número de profissionais, pois o interesse era de estabelecer um diagnóstico universal. Foram estabelecidos como critérios de exclusão um tempo de permanência na equipe inferior a três meses e atuação em equipes da zona rural.

Foram elaborados dois questionários: um para profissionais de nível superior (médicos e enfermeiros) e outro destinado a profissionais de nível médio (auxiliares de enfermagem e agentes comunitários de saúde). A maioria das questões apresentadas foi previamente testada em estudos semelhantes 7 . Os questionários foram pre- enchidos de forma individual e anônima em um mesmo momento, de forma a evitar que discussões posteriores sobre o tema interferissem nas respostas.

As perguntas abordadas nos questionários foram codificadas em variáveis categóricas. A comparação das proporções foi realizada por meio do teste do qui-quadrado ou teste exato de Fischer. Na interpretação dos resultados da avaliação do nível de conhecimento, o teste ANOVA foi utilizado na comparação das médias.

Os profissionais entrevistados concordaram com a realização da entrevista através de termo de consentimento livre e esclarecido. Este trabalho foi aprovado pelo Comitê de Ética em Pesquisa da Universidade Estadual de Montes Claros.

\section{Resultados}

Foram entrevistados 41 profissionais de nível superior e 152 de nível médio. O perfil dos profissionais estudados é apresentado na Tabela 1. Em relação à experiência pessoal com aleitamento materno, destacou-se o fato de que mais da me-

Características demográficas e experiências com aleitamento materno para profissionais da estratégia de saúde da família. Montes Claros, Minas Gerais, Brasil, 2005.

\begin{tabular}{|c|c|c|}
\hline \multirow[t]{3}{*}{ Característica } & \multicolumn{2}{|c|}{ Categoria profissional } \\
\hline & Médicos e enfermeiros & Auxiliares e agentes comunitários \\
\hline & $(n=41)$ & de saúde $(n=152)$ \\
\hline \multicolumn{3}{|l|}{ Idade } \\
\hline Amplitude & $23-42$ anos & $21-52$ anos \\
\hline Mediana & 29 anos & 30 anos \\
\hline \multicolumn{3}{|l|}{ Gênero } \\
\hline Masculino & $12(29,3 \%)$ & $27(17,8 \%)$ \\
\hline Feminino & $29(70,7 \%)$ & $125(82,2 \%)$ \\
\hline \multicolumn{3}{|c|}{ Numero de filhos } \\
\hline Nenhum & $25(61,0 \%)$ & $56(36,8 \%)$ \\
\hline $1-2$ & $13(31,7 \%)$ & $36(25,0 \%)$ \\
\hline 3 ou mais & $03(7,3 \%)$ & $58(38,2 \%)$ \\
\hline \multicolumn{3}{|c|}{ Experiência com aleitamento materno dos filhos } \\
\hline Não & $0(0,0 \%)$ & $12(7,9 \%)$ \\
\hline Sim & $16(100 \%)$ & $140(92,1 \%)$ \\
\hline \multicolumn{3}{|c|}{ Aleitamento materno exclusivo dos filhos (meses) } \\
\hline $0-2$ & $4(25,0 \%)$ & $19(20,2 \%)$ \\
\hline $2-4$ & $6(37,5 \%)$ & $33(35,1 \%)$ \\
\hline $5-6$ & $6(37,5 \%)$ & $42(44,7 \%)$ \\
\hline \multicolumn{3}{|c|}{ Tempo na equipe do Programa Saúde da Família } \\
\hline Amplitude & 4-48 meses & 4-86 meses \\
\hline Mediana & 24 meses & 44 meses \\
\hline
\end{tabular}


tade dos profissionais de saúde vivenciou uma interrupção precoce da amamentação exclusiva dos seus próprios filhos. A maioria dos entrevistados $(69,4 \%)$ referiu nunca ter realizado capacitação específica sobre amamentação.

A Tabela 2 apresenta o desempenho dos profissionais nos testes de conhecimento sobre aleitamento materno, com distribuição em quatro áreas temáticas. Observou-se um desempenho superior a $80 \%$ para as três categorias profissionais no aspecto de reconhecimento das vantagens do aleitamento materno. Em relação à técnica correta da amamentação, conhecimentos gerais sobre o leite materno, cuidados com a mama e manejo dos principais problemas, o melhor desempenho observado foi do grupo de enfermeiros. O grupo dos médicos e dos Agentes Comunitários de Saúde (ACS) apresentou desempenho médio abaixo de $50 \%$ para a área temática de técnica de amamentação e manejo dos principais problemas.

A Tabela 3 apresenta atividades regularmente desenvolvidas pela equipe das estratégias de saúde da família, na promoção do aleitamento materno. As ações de promoção do aleitamento materno não são desenvolvidas uniformemente. A orientação precoce para o aleitamento materno, a observação das mamadas e a realização de visitas puerperais precoces são ações mais comumente realizadas pelos auxiliares e agentes comunitários de saúde. De forma similar, a participação em grupos de gestantes ou nutrizes e a observação da mamada são atividades mais observadas entre os enfermeiros do que entre os médicos.

\section{Discussão}

O perfil dos profissionais avaliados (Tabela 1) é compatível com outros estudos sobre os recursos humanos para as estratégias de saúde da família. Destaca-se, assim, a relevância e a pertinência do tema diante da expansão da estratégia verificada nos últimos anos. A análise do desempenho nos testes de conhecimentos específicos (Tabela 2) mostrou que, quase sempre, os profissionais de saúde têm o conhecimento teórico das vantagens do aleitamento materno. A média superior a $80 \%$ para todas as categorias profissionais pode ser considerada satisfatória, quando comparada a outros estudos 7,8. Todavia, o desempenho abaixo de $50 \%$ para os médicos em relação à técnica da amamentação e ao manejo dos principais problemas da lactação mostra que tais profissionais não têm sustentação científica para abordar questões mais complexas e não podem oferecer adequado suporte às mães com alguma dificuldade em amamentar.

Ainda em relação à Tabela 2, nota-se que houve diferença estatisticamente significante entre o desempenho de médicos e enfermeiros nas áreas temáticas referentes à técnica da amamentação e cuidados com a mama. Esse fato denota, possivelmente, o descaso com a prática da amamentação nos cursos de graduação médica 5 . Mesmo com um teste mais fácil e com linguagem mais acessível, o desempenho dos ACS foi significativamente menor do que o dos profissionais de nível superior em três áreas temáticas. Essa falta de uniformidade entre os profissionais da ESF já foi apontada em outro estudo na região metropolitana de São Paulo ${ }^{8}$.

As atividades práticas que representam oportunidades de orientação, apoio e incenti-

\begin{tabular}{|c|c|c|c|c|c|}
\hline Áreas temáticas & $\begin{array}{c}\text { Médicos } \\
\begin{array}{c}(n=15) \\
\%\end{array}\end{array}$ & $\begin{array}{l}\text { Enfermeiros } \\
\qquad \begin{array}{c}(n=26) \\
\%\end{array}\end{array}$ & Valor de $p$ * & $\begin{array}{c}\text { ACS } \\
(n=152) \\
\%\end{array}$ & Valor de $p * *$ \\
\hline Vantagens do aleitamento materno & 83 & 86 & 0,251 & 85 & 0,672 \\
\hline Técnica correta à amamentação ** & 44 & 60 & 0,000 & 22 & 0,000 \\
\hline Conhecimentos gerais sobre o leite materno e cuidados com a mama ** & 70 & 82 & 0,000 & 55 & 0,000 \\
\hline Manejo dos principais problemas (ingurgitamento e fissuras) $\star \star$ & 42 & 48 & 0,071 & 32 & 0,000 \\
\hline
\end{tabular}

ACS: agentes comunitários de saúde.

* Comparação de desempenho nos testes cognitivos entre médicos e enfermeiros (teste ANOVA);

** Comparação de desempenho nos testes cognitivos entre profissionais de nível superior e nível médio (teste ANOVA). 
Atividades de promoção do aleitamento materno entre os profissionais das estratégias de saúde da família. Montes Claros, Minas Gerais, Brasil, 2005.

\begin{tabular}{|c|c|c|c|c|c|c|c|c|}
\hline \multirow[t]{2}{*}{ Atividades regulares de promoção do aleitamento materno } & \multicolumn{2}{|c|}{$\begin{array}{l}\text { Médicos } \\
(n=15)\end{array}$} & \multicolumn{2}{|c|}{$\begin{array}{l}\text { Enfermeiros } \\
\qquad(n=26)\end{array}$} & \multirow[t]{2}{*}{ Valor de $p^{*}$} & \multicolumn{2}{|c|}{$\begin{array}{c}\text { ACS } \\
(n=152)\end{array}$} & \multirow[t]{2}{*}{ Valor de $p^{\star \star}$} \\
\hline & $\mathbf{n}$ & $\%$ & n & $\%$ & & $\mathrm{n}$ & $\%$ & \\
\hline Orienta desde a fase inicial do pré-natal & 11 & 73,3 & 21 & 80,8 & 0,701 & 142 & 93,4 & 0,007 \\
\hline Orienta ao final do pré-natal & 12 & 80,0 & 23 & 88,4 & 0,651 & 134 & 88,2 & 0,830 \\
\hline Estimula a amamentação nas atividades de puericultura & 13 & 86,6 & 26 & 100,0 & 0,128 & 141 & 92,8 & 0,739 \\
\hline Avalia a mamada durante as visitas & 1 & 6,7 & 14 & 53,8 & 0,007 & 100 & 65,8 & 0,001 \\
\hline Orienta para prevenção de problemas com as mamas & 10 & 66,7 & 18 & 69,2 & 1,000 & 125 & 82,2 & 0,082 \\
\hline Realiza visitas puerperais precocemente & 4 & 26,7 & 15 & 57,7 & 0,707 & 132 & 86,8 & 0,000 \\
\hline Participa e orienta a amamentação em grupos de gestantes & 9 & 60,0 & 26 & 100,0 & 0,001 & 110 & 72,4 & 0,132 \\
\hline Participa de grupos de nutrizes & 4 & 26,7 & 23 & 88,4 & 0,000 & 110 & 72,4 & 0,534 \\
\hline Participa de grupos internos na unidade para discutir o tema & 6 & 40,0 & 12 & 46,2 & 0,955 & 78 & 51,3 & 0,505 \\
\hline Encaminha casos com dificuldade & 10 & 66,7 & 15 & 57,7 & 0,814 & 117 & 76,9 & 0,063 \\
\hline
\end{tabular}

ACS: agentes comunitários de saúde.

* Comparação entre as proporções de médicos e enfermeiros que realizam a atividade (teste do qui-quadrado ou teste exato de Fisher);

** Comparação entre as proporções de profissionais de nível superior e profissionais de nível médio que realizam a atividade (teste do qui-quadrado ou teste exato de Fisher).

vo ao aleitamento materno são apresentadas na Tabela 3. Orientações desde a fase inicial do pré-natal, a observação das mamadas durante os encontros e a realização precoce de visitas puerperais são atividades mais realizadas pelos ACS do que por outros profissionais $(\mathrm{p}<0,05)$. Entre os de nível superior, o enfermeiro realiza mais ações de promoção do que o médico, com diferenças estatisticamente significantes para as ações de observação da mamada, participação em grupos de gestantes e de nutrizes $(\mathrm{p}<0,05)$. De modo geral, as ações dos médicos podem ser consideradas tímidas e devem ser melhoradas a partir de uma postura mais ativa desses profissionais.

A opção por iniciar a amamentação é bastante comum para a maioria das mães, incluindo entre as da área em estudo ${ }^{9}$. Contudo, nem sempre essa sensibilização inicial é suficiente. A técnica da amamentação necessita de suporte adequado e contínuo para algumas mães. Se esse suporte não é disponível nas unidades básicas de saúde, inicia-se um processo de sofrimento materno fundamentado no ingurgitamento, nas fissuras e na percepção de fome através do comportamento do recém-nascido. De forma paralela, o discurso de "leite fraco" ou "leite ralo" amplamente difundido na população, acelera a introdução precoce de outros alimentos e culmina com o desmame. É natural concluir, diante dos resultados, que falta às mães o suporte adequado para correção da técnica e manejo adequado dos principais problemas, o que representaria uma atitude mais ativa e eficaz dos profissionais de saúde na promoção da amamentação. O simples fato de observar a mamada, atento à postura materna e à pega do recém-nascido ao seio materno, oferece subsídios importantes ao profissional de saúde sobre os riscos de desmame precoce. Contudo, essa prática é pouco comum entre médicos e enfermeiros (Tabela 3). Oportunidades de orientações precoces durante o pré-natal também são negligenciadas por uma parcela dos profissionais. Também chama a atenção a baixa participação do médico em atividades educativas coletivas (grupo de gestantes e nutrizes) e em visitas/consultas puerperais precoces, fato que pode comprometer o sucesso da amamentação, pela representação social do mesmo na população.

Os resultados revelam, enfim, que é possível melhorar o desempenho dos profissionais da estratégia de saúde da família em relação à amamentação, o que determina o compromisso social de sensibilizar e capacitar toda a equipe, sistematicamente. Essa tarefa torna-se fundamental diante de estudos que asseguram a importância e a efetividade de atividades de promoção do aleitamento materno em unidades de cuidado primário 10.

Embora tenham apresentado pior desempenho nos testes de conhecimentos, os ACS foram, no universo pesquisado, os que mais se declararam empenhados em atividades de promoção do 
aleitamento materno. É importante que programas específicos de capacitação incluam necessariamente esse grupo de profissionais, de forma a tornar mais efetiva e significativa a participação dos mesmos na melhoria dos índices de aleitamento materno.

\section{Resumo}

A estratégia de saúde da família foi implantada no Brasil buscando reorganizar a assistência à saúde, com ênfase em ações de promoção e prevenção. Assim, o incentivo ao aleitamento materno é uma das atividades mais importantes para as equipes de saúde da família. Este estudo objetivou avaliar conhecimentos e práticas das referidas equipes em relação ao aleitamento materno. Além de um questionário para definição do perfil dos profissionais, um teste de conhecimentos sobre amamentação foi aplicado a 41 médicos e enfermeiros, e outro teste sobre o mesmo tema foi aplicado para 152 agentes comunitários de saúde. O desempenho das equipes sobre vantagens do aleitamento materno mostrou-se superior a 80\%, mas o conhecimento sobre questões relacionadas à técnica da amamentação e manejo dos problemas relacionados à lactação não foi uniforme entre os profissionais. Enfermeiros apresentaram melhor desempenho nos testes e agentes de saúde tiveram as piores pontuações. Atividades práticas de promoção do aleitamento materno não eram desenvolvidas uniformemente pelos profissionais. Há necessidade de treinamento sistemático e contínuo sobre aleitamento materno e manejo da lactação para equipes de saúde da família.

Aleitamento Materno; Programa Saúde da Família; Atenção Primária à Saúde; Promoção da Saúde

\section{Colaboradores}

A. P. Caldeira participou em todas as fases do trabalho. W. A. C. Magalhães participou do processo de coleta, codificação e análise dos dados e contribuiu na elaboração do texto final. G. N. Aguiar e G. C. Fagundes participaram da coleta e processamento de dados, da análise e interpretação dos resultados e da redação do texto final.

\section{Agradecimentos}

Este trabalho contou com o financiamento da Fundação de Amparo à Pesquisa do Estado de Minas Gerais (Processo ${ }^{\circ}$. EDT-130/05). 


\section{Referências}

1. Victora CG, Vaughan JP, Lombardi C, Fuchs SMC, Gigante LP, Smith PG, et al. Evidence for protection by breast-feeding against infant deaths from infectious diseases in Brazil. Lancet 1987; 2:31922.

2. Victora CG, Fuchs SC, Flores JAC, Fonseca W, Kirkwood B. Risk factors for pneumonia among children in a brazilian metropolitan area. Pediatrics 1994; 93:977-85.

3. Oddy WH. Breastfeeding protects against illness and infection in infants and children: a review of the evidence. Breastfeed Rev 2001; 9:11-8.

4. Organização Mundial da Saúde. Proteção, promoção e apoio ao aleitamento materno: o papel especial dos serviços materno-infantis. Genebra: Organização Mundial da Saúde; 1989.

5. Krogstrand KS, Parr K. Physicians ask for more problem-solving information to promote and support breastfeeding. J Am Diet Assoc 2005; 105:19437.

6. Oliveira MI, Camacho LA, Tedstone AE. A method for the evaluation of primary health care units' practice in the promotion, protection, and support of breastfeeding: results from the state of Rio de Janeiro, Brazil. J Hum Lact 2003; 19:365-73.
7. Becker D. No seio da família: amamentaçäo e promoçäo da saúde no Programa de Saúde da Família [Dissertação de Mestrado]. Rio de Janeiro: Escola Nacional de Saúde Pública, Fundação Oswaldo Cruz; 2001.

8. Ciconi RCV, Venâncio SI, Escuder MML. Avaliação dos conhecimentos de equipes do Programa de Saúde da Família sobre o manejo do aleitamento materno em um município da região metropolitana de São Paulo. Rev Bras Saúde Matern Infant 2004; 4:193-202.

9. Caldeira AP, Goulart EMA. A situação do aleitamento materno em Montes Claros, Minas Gerais: estudo de uma amostra representativa. J Pediatr (Rio de J) 2000; 76:65-72.

10. Oliveira MIC, Camacho LAB, Tedstone AE. Extending breastfeeding duration through primary care: a systematic review of prenatal and postnatal interventions. J Hum Lact 2001; 17:326-43.

Recebido em 22/Mai/2006

Versão final reapresentada em 6/Mar/2007 Aprovado em 19/Abr/2007 